\title{
Prediction of the Remnant Liver Hypertrophy Ratio after Preoperative Portal Vein Embolization
}

\author{
Y. Kasai ${ }^{a} \quad$ E. Hatano ${ }^{a} \quad$ K. Iguchi ${ }^{a} \quad$ S. Seo ${ }^{a} \quad$ K. Taura ${ }^{a} \quad$ K. Yasuchika ${ }^{a}$
T. Kaido ${ }^{a}$
S. Tanakac
T. Shibatab
T. Shibatab
S. Uemoto ${ }^{a}$ \\ A. Moria
}

Departments of a Surgery and ${ }^{b}$ Diagnostic Imaging and Nuclear Medicine, Graduate School of Medicine, and ' Department of Pharmacoepidemiology, Graduate School of Medicine and Public Health, Kyoto University, Kyoto, Japan

\section{Key Words}

Portal vein embolization · Hypertrophy ratio · Prediction · Major hepatectomy · Associating liver partition and portal vein ligation for staged hepatectomy

\begin{abstract}
Background: Portal vein embolization (PVE) is considered to improve the safety of major hepatectomy. Various conditions might affect remnant liver hypertrophy after PVE. The aim of the present study was to clarify the factors that affect remnant liver hypertrophy and to establish a prediction formula for the hypertrophy ratio. Methods: Fifty-nine patients who underwent preoperative PVE for cholangiocarcinoma (39 patients), metastatic carcinoma (10 patients), hepatocellular carcinoma (8 patients), and other diseases (2 patients) were enrolled in this study. For the prediction of the hypertrophy ratio, a formula with stepwise multiple regression analysis was set up. The following parameters were used: age, gender, future liver remnant ratio to total liver (FLR\%), plasma disappearance rate of indocyanine green (ICGK), platelet count, prothrombin activity, serum albumin, serum total bilirubin at the time of PVE and the maximum value before PVE (Max Bil), as well as a history of cholangitis, diabetes mellitus, and chemotherapy. Results: The mean hypertrophy ratio was $28.8 \%$. The 5 parameters detected as predictive factors were age $(p=0.015)$, FLR\% $(p<0.001)$, ICGK $(p=0.112)$, Max Bil $(p<0.001)$, and history of chemotherapy $(p=0.007)$. The following prediction formula was established: $101.6-0.78 \times$ age $-0.88 \times \mathrm{FLR} \%+128 \times \mathrm{ICGK}-1.48 \times \mathrm{Max} \mathrm{Bil}(\mathrm{mg} / \mathrm{dl})-21.2 \times$ chemotherapy. The value obtained using this formula significantly correlated with the actual value $(r=0.72, p<0.001)$. A 10 -fold cross validation also showed significant correlation $(r=$ $0.62, p<0.001$ ), and a hypertrophy ratio $<20 \%$ was predictable with a sensitivity of $100 \%$ and a specificity of $90.9 \%$. Moreover, technetium-99m-diethylenetriaminepentaacetic acid-galac-
\end{abstract}


Kasai et al.: Prediction of the Remnant Liver Hypertrophy Ratio after Preoperative Portal Vein Embolization

tosyl human serum albumin scintigraphy showed a significantly smaller increase in the uptake ratio of the remnant liver in patients with prediction values $<20 \%$ than in those with values $\geq 20 \%$ (6.8 vs. $20.8 \%, p=0.030$ ). Conclusions: The prediction formula can prognosticate the hypertrophy ratio after PVE, which may provide a new therapeutic strategy for major hepatectomy.

(C) 2013 S. Karger AG, Basel

\section{Introduction}

Liver resection is usually the only radical therapy for primary and metastatic liver tumor except for liver transplantation. Although liver surgery has become much safer because of improved diagnostic imaging, surgical procedures, and perioperative management during the past decades [1], the problems of high mortality and morbidity rates in major hepatectomy remain unsolved. Particularly, post-hepatectomy liver failure is a lethal complication [2]. The future liver remnant (FLR) volume has to be $>30 \%$ in normal livers and $50 \%$ in damaged livers in order to avoid liver failure [3]. In cases with an insufficient FLR volume, portal vein embolization (PVE) generally has been performed to induce compensatory hypertrophy of the remnant liver and to increase safety of major hepatectomy [4]. Previous PVE studies demonstrated that FLR volume increased by 20-50\% within a 3- to 7-week interval between PVE and hepatectomy [5-8]. However, various conditions, including hepatitis, cholestasis, and chemotherapy, are reported to affect remnant liver hypertrophy [7, 9-12]. Meanwhile, 10-20\% of the cases become unresectable after such an interval because of tumor progression or insufficient remnant liver hypertrophy $[13,14]$.

Recently, Schnitzbauer et al. [15] reported a novel strategy of associating liver partition and portal vein ligation for staged hepatectomy (ALPPS), which induced marked and rapid remnant liver hypertrophy. Although other studies have demonstrated its usefulness [1618], the indication for ALPPS should be restricted because of the complexity of the surgical procedure and the high morbidity rate.

Early prediction of the hypertrophy ratio after PVE may enable the identification of patients who will be refractory to PVE and may be useful in determining the indication for ALPPS. Therefore, the aim of the present study was to clarify the factors that affect remnant liver hypertrophy after PVE and to establish a prediction formula for the hypertrophy ratio.

\section{Methods}

Study Design

We performed a retrospective cohort study of patients with liver diseases who underwent preoperative PVE between January 2005 and December 2012 at the Department of Surgery, Kyoto University Hospital. In total, PVE was performed in 65 patients. Six patients were excluded from the analysis either because they underwent embolization of the portal branches of $\leq 1$ segment $(n=5)$ or the post-PVE CT scan was not performed because exploratory laparoscopy revealed peritoneal dissemination $(n=1)$. After these exclusions, 59 patients were analyzed (fig. 1), and the diseases were cholangiocarcinoma in 39 patients (66\%), metastatic carcinoma in 10 patients (17\%), hepatocellular carcinoma in 8 patients $(14 \%)$, cystic tumor of the liver in 1 patient, and benign gallbladder tumor in 1 patient. This study was in accordance with the ethical guidelines for epidemiological research in Japan and had been approved by the Ethics Committee of the Kyoto University Graduate School and Faculty of Medicine (approval code: E1737).

Indication for PVE and Procedure

The indication for PVE was as follows: future liver remnant ratio to total liver (FLR\%) $<30 \%$, planned hepatopancreatoduodenectomy, or poor functional reserve [3]. PVE was routinely performed by the percu- 
Kasai et al.: Prediction of the Remnant Liver Hypertrophy Ratio after Preoperative Portal Vein Embolization

Fig. 1. Study population, surgical procedures, and the reasons for unresectability after PVE. HPD = Hepatopancreatoduodenectomy.
65 patients underwent PVE between 2005 and 2012

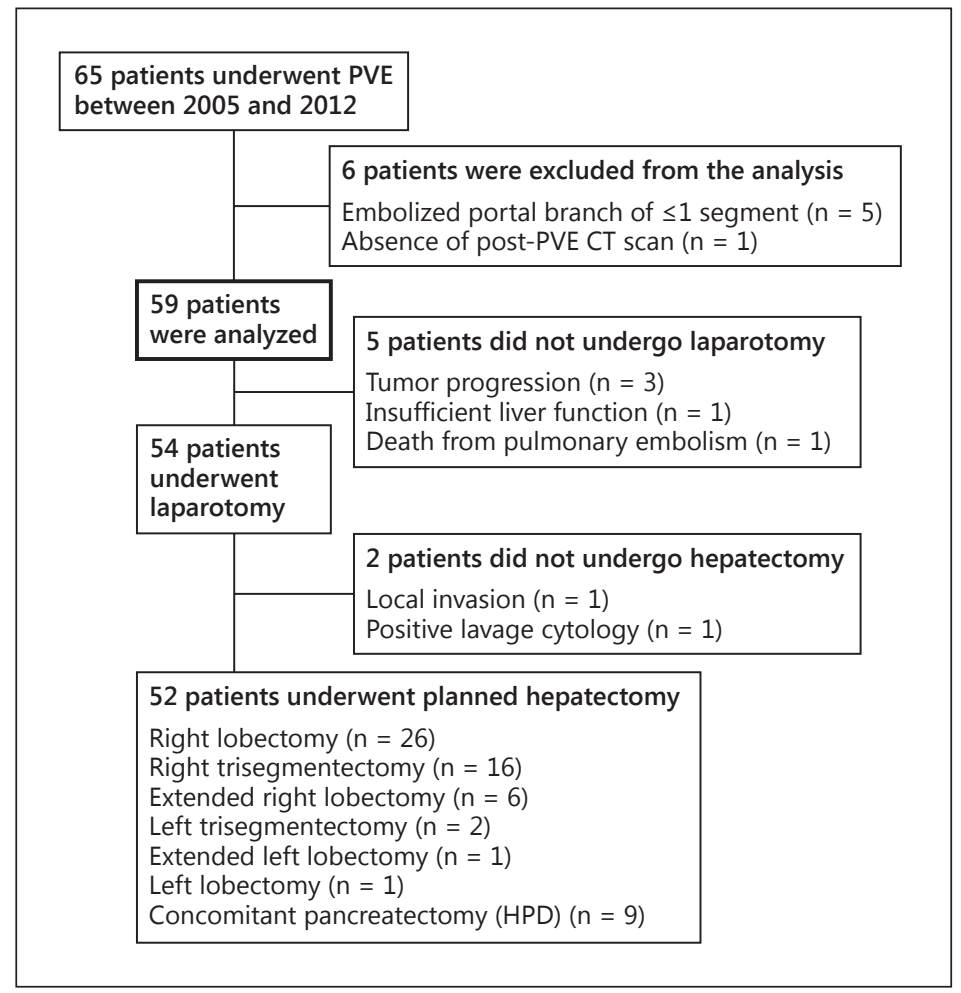

taneous transhepatic ipsilateral approach, while the contralateral approach was only used in patients for whom the ipsilateral approach was unsuitable. The embolization materials consisted of absolute ethanol (dehydrated ethanol; Mylan, Tokyo, Japan), iodized oil (Lipiodol Ultra-Fluide; Terumo, Tokyo, Japan), and porous gelatin particles (Gelpart; Nippon Kayaku, Tokyo, Japan) with or without detachable microcoils (Presidio and Cashmere; Codman Johnson and Johnson, New Brunswick, N.J., USA). The embolized portal branches were the right branch in 47 patients $(80 \%)$, the right branch with the branch of segment IV in 8 patients (14\%), the left branch in 1 patient (2\%), and the left branch with the anterior branch in 3 patients (5\%).

\section{CT Volumetry}

Multislice CT scans were performed before and 3 weeks after PVE. The liver volume was measured by cutting the liver in slices of 1-mm thickness each on a workstation (AZE VirtualPlace Plus; AZE, Tokyo, Japan). FLR\% and hypertrophy ratio were calculated using the following formulas:

FLR\% $=100 \times$ FLR $(\mathrm{ml}) /[$ total liver volume $(\mathrm{ml})-$ tumor volume $(\mathrm{ml})]$ and

hypertrophy ratio $(\%)=100 \times[$ FLR after PVE $(\mathrm{ml})-$ FLR before PVE $(\mathrm{ml})] /$ FLR before PVE $(\mathrm{ml})$.

\section{Predictive Model for the Hypertrophy Ratio}

Predictive factors for the hypertrophy ratio were detected by a stepwise variable selection based on Bayesian information criteria among the following variables: age, gender, FLR\%, plasma disappearance rate of indocyanine green (ICGK), platelet count, prothrombin activity, serum albumin, serum total bilirubin at the time of PVE (Bil at PVE) and the maximum value before PVE (Max Bil), as well as a history of cholangitis, diabetes mellitus, and $\geq 6$ cycles of systemic chemotherapy. Then, the prediction formula for the hypertrophy ratio was set up with the detected predictive factors by multiple regression analysis. The male and female gender was defined as ' 1 ' and ' 0 ', respectively. History of cholangitis, diabetes mellitus, and chemotherapy was defined as ' 1 ' if there was a history and as ' 0 ' if there was no history. The ICG test was performed under the condition of serum total bilirubin $<5 \mathrm{mg} / \mathrm{dl}$ in all cases.

After detection of the predictive factors, a 10 -fold cross validation was performed for the predictive model. In brief, all patients were randomly divided into 10 groups. The prediction value of the hypertrophy 
Fig. 2. FLR volume change after

Kasai et al.: Prediction of the Remnant Liver Hypertrophy Ratio after Preoperative Portal Vein Embolization

PVE.

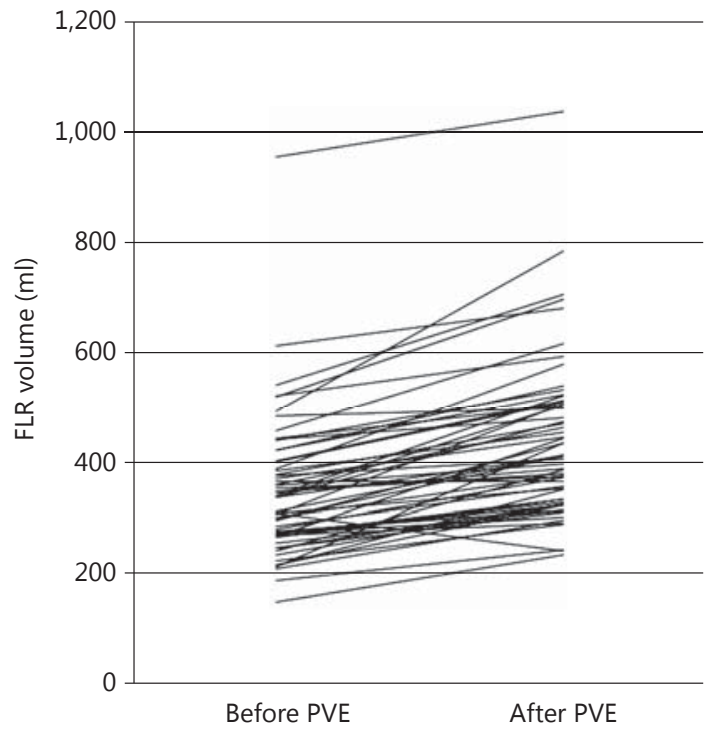

ratio in a group was calculated with the prediction formula set up by multiple regression analysis of the other 9 groups, using the detected predictive factors. This calculation was repeated, and then we evaluated calibration and discriminatory power of the predictive model. We constructed scatter plots of predicted and observed hypertrophy ratios to evaluate calibration and used sensitivity and specificity to identify patients with a hypertrophy ratio $<20 \%$ as a criterion for discriminatory power.

Functional volume change was analyzed by technetium-99m-diethylenetriaminepentaacetic acidgalactosyl human serum albumin ( $\left.{ }^{99 \mathrm{~m}} \mathrm{Tc}-\mathrm{GSA}\right)$ scintigraphy to examine whether the prediction formula could reflect functional gain.

\section{Statistical Analysis}

Data were expressed as mean \pm SD unless otherwise indicated. Volumetric differences before and after PVE were compared using the paired test, while other continuous variables were compared using the unpaired t test. A two-tailed p value $<0.05$ was considered significant. All analyses were performed using JMP software version 10 (SAS Institute Inc., Cary, N.C., USA).

\section{Results}

\section{Patients' Clinical Course after PVE}

Most patients experienced transient fever after PVE. Two patients experienced complicated hemorrhagic events (subcapsular hematoma and intra-abdominal hemorrhage) that were managed conservatively, and 1 case was complicated by bile leakage managed with percutaneous drainage. FLR volume significantly increased from $346 \pm 126 \mathrm{ml}(29.2 \pm 9.5 \%)$ to $438 \pm 144 \mathrm{ml}(37.5 \pm 10.6 \%, \mathrm{p}<0.001)$ in 3 weeks (median: 21 days, range: $14-99$ days) after PVE. The mean hypertrophy ratio was $28.8 \pm 20.7 \%$ (range: -22.0 to 105.1) (fig. 2). Of those undergoing PVE, 5 patients did not undergolaparotomy because of (a) tumor progression (3 patients); (b) insufficient liver function (1 patient), and (c) death from pulmonary embolism (1 patient). Fifty-two patients (88\%) underwent planned hepatectomy, while the 2 patients with hilar cholangiocarcinoma underwent exploratory laparotomy because of local invasion in 1 patient and positive lavage cytology in the other (fig. 1). 
Table 1. Multiple regression analysis

\begin{tabular}{llccr}
\hline Variables & Mean $^{\mathrm{a}}$ & $\beta$ & $95 \% \mathrm{CI}(\mathrm{L}, \mathrm{U})$ & \multicolumn{1}{c}{ p value } \\
\hline Age, years & $65.3 \pm 9.5$ & -0.78 & $-1.40,-0.16$ & 0.015 \\
FLR\% & $29.2 \pm 9.5$ & -0.88 & $-1.36,-0.41$ & $<0.001$ \\
ICGK & $0.14 \pm 0.03$ & 128 & $-31.2,288$ & 0.112 \\
Max Bil, mg/dl & $7.37 \pm 8.95$ & -1.48 & $-2.23,-0.73$ & $<0.001$ \\
Chemotherapy (yes/no) & $7 / 52$ & -21.2 & $-36.1,-6.28$ & 0.007 \\
\hline
\end{tabular}

$\beta=$ Regression coefficient; $95 \% \mathrm{CI}=95 \%$ confidence interval; $\mathrm{L}=$ lower confidence limit; $\mathrm{U}=$ upper confidence limit.

${ }^{\text {a }}$ Values are means $\pm \mathrm{SD}$, unless otherwise specified.

\section{Predictive Factors and Prediction Formula}

The mean age was $65.3 \pm 9.5$ years (range: $33-84$ ), and 39 patients were male and 20 were female. Thirty-eight patients (64\%) underwent biliary drainage for obstructive cholestasis, and 15 patients $(25 \%)$ had the complication of cholangitis. Seven patients with colorectal liver metastasis received systemic chemotherapy with a median of 11 cycles (range: 6-29) of the oxaliplatin or irinotecan regimen. In simple regression analysis, FLR\% ( $p=$ $0.010)$, ICGK $(\mathrm{p}=0.018)$, Bil at PVE $(\mathrm{p}=0.028)$ and Max Bil $(\mathrm{p}=0.045)$ were significant factors. In stepwise variable selection, the following 5 parameters were detected as predictive factors: ICGK $(p=0.112)$ as a positive factor and age $(p=0.015)$, FLR $\%(p<0.001)$, Max Bil $(p<0.001)$, and history of chemotherapy $(p=0.007)$ as negative factors (table 1$)$. The following prediction formula was calculated:

$101.6-0.78 \times$ age $-0.88 \times$ FLR\% $+128 \times$ ICGK $-1.48 \times$ Max Bil (mg/dl) $-21.2 \times$ chemotherapy

The value obtained using this formula significantly correlated with the actual value $(\mathrm{r}=$ 0.72 , p < 0.001) (fig. 3a). When the cutoff value was set at $20 \%$, hypertrophy ratio values $<20 \%$ were predictable with a sensitivity of $100 \%$ and a specificity of $90.9 \%$. Significant correlation was also observed in each of the following diseases: cholangiocarcinoma $(r=0.68$, $p<0.001)$, metastatic carcinoma $(r=0.93, p<0.001)$, and hepatocellular carcinoma $(r=0.78$, $\mathrm{p}=0.040$ ). A 10 -fold cross validation also showed significant correlation between the observed and predicted values $(r=0.62, \mathrm{p}<0.001)$, and the slope of the regression line was 0.87 , indicating that the calibration of the prediction formula was good (fig. 3b). A hypertrophy ratio $<20 \%$ in the 10 -fold cross validation was predictable with a sensitivity of $100 \%$ and a specificity of $90.9 \%$.

Three outliers in the prediction of the hypertrophy ratio with values either $<20 \%$ or $\geq 20 \%$ are shown in table 2 . All 3 patients presented a much smaller hypertrophy ratio than predicted, and 2 of the 3 patients experienced complications of PVE, intra-abdominal hemorrhage and bile leakage, which might be the causes of poor response to PVE.

${ }^{99 \mathrm{~m}}$ Tc-GSA scintigraphy was performed both before and after PVE in 20 patients, 7 of them with prediction values $<20 \%$ and 13 with values $\geq 20 \%$. The increase in the uptake ratio of the remnant liver (after PVE - before PVE) was significantly lower in patients with prediction values $<20 \%$ than in those with values $\geq 20 \%$ ( $6.8 \pm 4.2$ vs. $20.8 \pm 15.3, p=0.030$; fig. 4). These results indicated that the formula established in this study could predict the degree of liver hypertrophy in an appropriate manner, reflecting the result of the ${ }^{99 \mathrm{~m}} \mathrm{Tc}-\mathrm{GSA}$ scintigraphy, and that it might be useful for clinical application. 


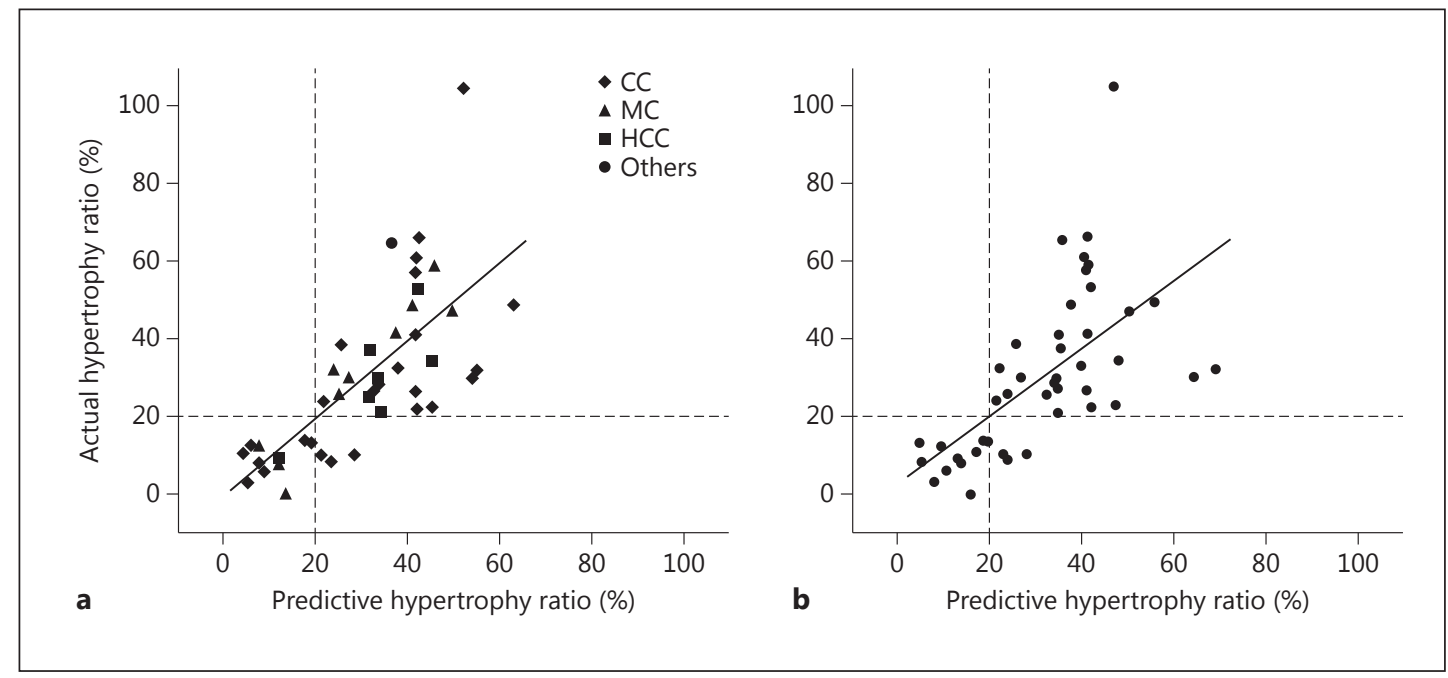

Fig. 3. a Correlation between predictive and actual hypertrophy ratio . Predictive hypertrophy ratio was significantly correlated with actual hypertrophy ratio $(r=0.72, p<0.001)$. A hypertrophy ratio $<20 \%$ was predictable with a sensitivity of $100 \%$ and a specificity of $90.9 \%$. Significant correlation with each of the following diseases was also observed: cholangiocarcinoma ( $\mathrm{r}=0.68, \mathrm{p}<0.001)$, metastatic carcinoma $(\mathrm{r}=0.93$, $\mathrm{p}<0.001)$, and hepatocellular carcinoma $(\mathrm{r}=0.78, \mathrm{p}=0.040)$. $\mathbf{b} \mathrm{A} 10$-fold cross validation also showed significant correlation $(\mathrm{r}=0.62, \mathrm{p}<0.001)$, and the slope of the regression line was 0.87 . CC $=$ Cholangiocarcinoma; $\mathrm{MC}=$ metastatic carcinoma; HCC = hepatocellular carcinoma.

Table 2. Outliers in the prediction

\begin{tabular}{|c|c|c|c|c|c|c|c|c|c|}
\hline $\begin{array}{l}\text { Age, } \\
\text { years }\end{array}$ & Gender & Diagnosis & FLR\% & ICGK & $\begin{array}{l}\text { Max Bil, } \\
\text { mg/dl }\end{array}$ & Chemo & $\begin{array}{l}\text { PHR, } \\
\%\end{array}$ & $\begin{array}{l}\text { AHR, } \\
\%\end{array}$ & Remarks \\
\hline 70 & $\mathrm{~F}$ & hilar CC & 21 & 0.149 & 13.4 & no & 27.5 & 10.4 & nonmedicated diabetes \\
\hline 74 & M & ICC & 27 & 0.117 & 8.2 & no & 22.9 & 8.9 & bile leakage \\
\hline 84 & $\mathrm{~F}$ & hilar CC & 33 & 0.127 & 1.7 & no & 20.7 & 10.4 & intra-abdominal hemorrhage \\
\hline
\end{tabular}

Chemo = Chemotherapy; $\mathrm{PHR}=$ predictive hypertrophy ratio; $\mathrm{AHR}=$ actual hypertrophy ratio; $\mathrm{CC}=$ cholangiocarcinoma; ICC = intrahepatic cholangiocarcinoma.

\section{Discussion}

In the present study, we analyzed a prediction formula for the hypertrophy ratio after PVE and explored the possibility of its application to a new strategy for hepatobiliary surgery. There have been several reports on the prediction of remnant liver hypertrophy after PVE. Imamura et al. [11] showed that diabetes mellitus, a high bilirubin level at the time of PVE, male gender, and FLR volume were the negative factors for remnant liver hypertrophy. A large FLR means a small volume of embolized liver parenchyma, and thus less impact on volume shift. Several studies, including ours, supported this finding $[7,19,20]$. Cholestasis is also a known inhibitor of hepatic regeneration [10], and Imamura et al. [11] emphasized the influence of the bilirubin level at the time of PVE on FLR hypertrophy. However, we first demonstrated the significance of the maximum bilirubin level before biliary drainage as a negative predictive factor for the hypertrophy ratio, which suggested that once exposed to 


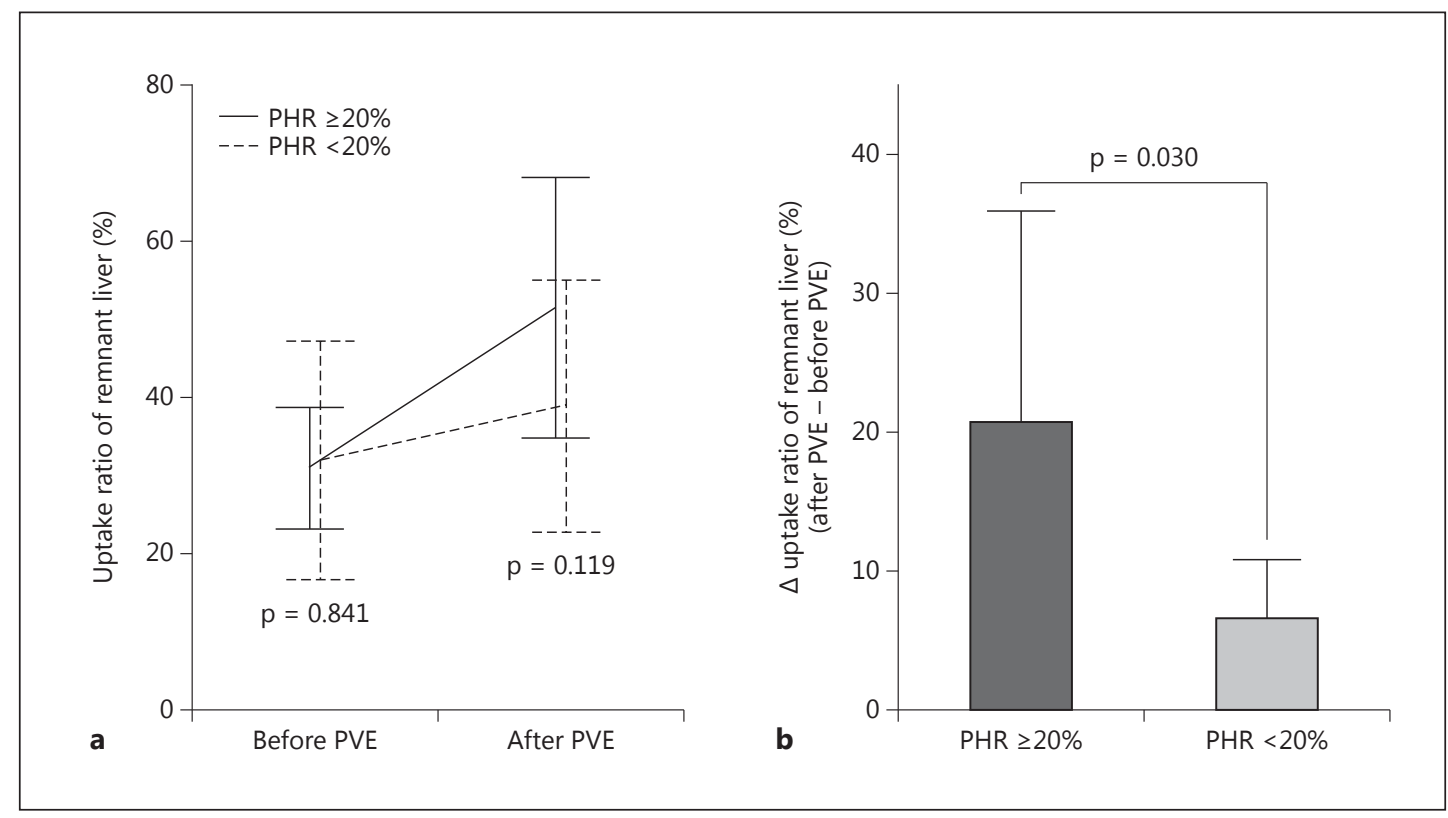

Fig. 4. Change in the uptake ratio of ${ }^{99 \mathrm{~m}} \mathrm{Tc}-\mathrm{GSA}$ scintigraphy. a Uptake ratio before and after PVE. There was no significant difference in the uptake ratio at baseline and after PVE between patients with a predictive hypertrophy ratio $</ \geq 20 \%$. b Increase in the uptake ratio after PVE. Patients with a predictive hypertrophy ratio $<20 \%$ gained a smaller functional volume shift than those with a ratio $\geq 20 \%$ (6.8 \pm 4.2 vs. $20.8 \pm 15.3 \%$, $\mathrm{p}=0.030$ ). PHR $=$ Predictive hypertrophy ratio.

high levels of cholestasis, the liver had attenuated regenerative capacity even if followed by adequate biliary drainage at the time of PVE. Nevertheless, best available biliary drainage should be performed before PVE, as portal flow occlusion under high levels of cholestasis might cause severe liver failure owing to the enhancement of hepatocyte apoptosis [10].

Novel chemotherapeutic drugs against colorectal cancer such as oxaliplatin and irinotecan have increased the pool of patients indicated for liver resection, which has augmented the need for PVE including 2-staged hepatectomy [21]. Meanwhile, oxaliplatin and irinotecan were reported to induce severe liver injury known as sinusoidal obstruction syndrome and steatohepatitis, respectively, and to impair liver regeneration [12, 22, 23]. Additionally, in the present study, a history of chemotherapy containing these drugs was indicated as a strong negative predictive factor (regression coefficient: -21.2). Although we could not clarify the association between the pathological findings of these liver injuries and the hypertrophy ratio because of the small number of patients with liver metastasis, liver biopsy might be considered before PVE to confirm the severity of liver injury in patients receiving multiple chemotherapy cycles.

Regarding the safety of major hepatectomy, functional as well as morphologic volume of FLR is an important matter. In ${ }^{99 \mathrm{~m}} \mathrm{Tc}$-GSA scintigraphy, the uptake ratio reflects a separate functional volume and is suited for the evaluation of functional volume shift after PVE [24]. Our data indicated that the liver with a lower prediction value of the hypertrophy ratio gained a smaller liver functional reserve, which was consistent with the idea that the prediction of morphologic volume change after PVE also reflects functional volume change. Moreover, this finding indicated that another imaging modality validated the prediction formula for the hypertrophy ratio measured by a CT scan. 
Recently, several authors have reported that ALPPS induced marked and rapid remnant liver hypertrophy and could reduce the risk of post-hepatectomy liver failure [15-18]. This novel technique is expected to be a breakthrough in the field of hepatobiliary surgery. However, ALPPS is a complex surgical procedure with a high morbidity rate, including bile leakage [15]. Therefore, the indication should be restricted. The proposed indication for ALPPS is bilateral liver metastasis, a very small FLR of $<25 \%$, or salvage for poor response to PVE $[18,25]$. Knoefel et al. [18] demonstrated that ALPPS for patients with poor response to PVE induced comparable FLR hypertrophy to those who underwent direct ALPPS without PVE, indicating the effectiveness of salvage ALPPS for poor responders to PVE. However, as PVE was reported to accelerate tumor proliferation during the waiting time [20], useless PVE should be avoided. Our prediction formula could prognosticate both morphologic and functional liver volume changes, especially in poor responders to PVE. Because poor response to PVE is regarded as a risk factor for post-hepatectomy liver failure $[7,26]$, those patients might need additional FLR volume to avoid liver failure. Therefore, patients with lower predictive hypertrophy ratios, who would gain insufficient FLR volume both morphologically and functionally if PVE was performed, might benefit from direct ALPPS without PVE. This strategy might enable those patients to achieve sufficient FLR hypertrophy without tumor progression during the waiting period.

There are several limitations in the present study. First, the sample size was relatively small. Second, we could not find evidence of a relationship between the predictive hypertrophy ratio and postoperative outcomes. Postoperative outcome also depends on underlying liver disease and subsequent liver damage, lymphadenectomy, biliary or vascular reconstruction, and concomitant pancreatectomy. Therefore, we considered it inadequate to unconditionally associate the prediction of the hypertrophy ratio with postoperative outcome, as we studied a heterogeneous population. Third, prospective or external validation is needed to further verify the prediction formula for application in clinical practice.

In conclusion, we demonstrated that a prediction formula could prognosticate the hypertrophy ratio after PVE, and FLR volume could be calculated with this formula. This may provide a new therapeutic strategy for major hepatectomy including ALPPS.

\section{Disclosure Statement}

The authors declare no conflicts of interest.

\section{References}

- 1 Miyagawa S, Makuuchi M, Kawasaki S, Kakazu T: Criteria for safe hepatic resection. Am J Surg 1995;169:589594.

2 Rahbari NN, Garden OJ, Padbury R, Brooke-Smith M, Crawford M, Adam R, Koch M, Makuuchi M, Dematteo RP, Christophi C, Banting S, Usatoff V, Nagino M, Maddern G, Hugh TJ, Vauthey JN, Greig P, Rees M, Yokoyama Y, Fan ST, Nimura Y, Figueras J, Capussotti L, Büchler MW, Weitz J: Posthepatectomy liver failure: a definition and grading by the International Study Group of Liver Surgery (ISGLS). Surgery 2011;149:713-724.

3 Clavien PA, Petrowsky H, DeOliveira ML, Graf R: Strategies for safer liver surgery and partial liver transplantation. N Engl J Med 2007;356:1545-1559.

- 4 Makuuchi M, Thai BL, Takayasu K, Takayama T, Kosuge T, Gunvén P, Yamazaki S, Hasegawa H, Ozaki H: Preoperative portal embolization to increase safety of major hepatectomy for hilar bile duct carcinoma: a preliminary report. Surgery 1990;107:521-527.

5 Liu H, Zhu S: Present status and future perspectives of preoperative portal vein embolization. Am J Surg 2009; 197:686-690.

6 Farges O, Belghiti J, Kianmanesh R, Regimbeau JM, Santoro R, Vilgrain V, Denys A, Sauvanet A: Portal vein embolization before right hepatectomy: prospective clinical trial. Ann Surg 2003;237:208-217. 
Kasai et al.: Prediction of the Remnant Liver Hypertrophy Ratio after Preoperative Portal Vein Embolization

7 Wakabayashi H, Ishimura K, Okano K, Karasawa Y, Goda F, Maeba T, Maeta H: Application of preoperative portal vein embolization before major hepatic resection in patients with normal or abnormal liver parenchyma. Surgery 2002;131:26-33.

- 8 Aussilhou B, Lesurtel M, Sauvanet A, Farges O, Dokmak S, Goasguen N, Sibert A, Vilgrain V, Belghiti J: Right portal vein ligation is as efficient as portal vein embolization to induce hypertrophy of the left liver remnant. J Gastrointest Surg 2008;12:297-303.

9 Tanaka H, Hirohashi K, Kubo S, Ikebe T, Tsukamoto T, Hamba H, Shuto T, Wakasa K, Kinoshita H: Influence of histological inflammatory activity on regenerative capacity of liver after percutaneous transhepatic portal vein embolization. J Gastroenterol 1999;34:100-104.

10 Yokoyama Y, Nagino M, Nimura Y: Mechanism of impaired hepatic regeneration in cholestatic liver. J Hepatobiliary Pancreat Surg 2007;14:159-166.

11 Imamura H, Shimada R, Kubota M, Matsuyama Y, Nakayama A, Miyagawa S, Makuuchi M, Kawasaki S: Preoperative portal vein embolization: an audit of 84 patients. Hepatology 1999;29:1099-1105.

-12 Narita M, Oussoultzoglou E, Chenard MP, Rosso E, Casnedi S, Pessaux P, Bachellier P, Jaeck D: Sinusoidal obstruction syndrome compromises liver regeneration in patients undergoing two-stage hepatectomy with portal vein embolization. Surg Today 2011;41:7-17.

13 Nagino M, Kamiya J, Nishio H, Ebata T, Arai T, Nimura Y: Two hundred forty consecutive portal vein embolizations before extended hepatectomy for biliary cancer: surgical outcome and long-term follow-up. Ann Surg 2006;243:364-372.

14 Abulkhir A, Limongelli P, Healey AJ, Damrah O, Tait P, Jackson J, Habib N, Jiao LR: Preoperative portal vein embolization for major liver resection: a meta-analysis. Ann Surg 2008;247:49-57.

15 Schnitzbauer AA, Lang SA, Goessmann H, Nadalin S, Baumgart J, Farkas SA, Fichtner-Feigl S, Lorf T, Goralcyk A, Hörbelt R, Kroemer A, Loss M, Rümmele P, Scherer MN, Padberg W, Königsrainer A, Lang H, Obed A, Schlitt HJ: Right portal vein ligation combined with in situ splitting induces rapid left lateral liver lobe hypertrophy enabling 2-staged extended right hepatic resection in small-for-size settings. Ann Surg 2012;255:405-414.

16 de Santibañes E, Alvarez FA, Ardiles V: How to avoid postoperative liver failure: a novel method. World J Surg 2012;36:125-128.

17 Alvarez FA, Ardiles V, Sanchez Claria R, Pekolj J, de Santibañes E: Associating liver partition and portal vein ligation for staged hepatectomy (ALPPS): tips and tricks. J Gastrointest Surg 2013;17:814-821.

-18 Knoefel WT, Gabor I, Rehders A, Alexander A, Krausch M, Schulte am Esch J, Fürst G, Topp SA: In situ liver transection with portal vein ligation for rapid growth of the future liver remnant in two-stage liver resection. Br J Surg 2013;100:388-394.

19 de Baere T, Teriitehau C, Deschamps F, Catherine L, Rao P, Hakime A, Auperin A, Goere D, Elias D, Hechelhammer L: Predictive factors for hypertrophy of the future remnant liver after selective portal vein embolization. Ann Surg Oncol 2010;17:2081-2089.

20 Kokudo N, Tada K, Seki M, Ohta H, Azekura K, Ueno M, Ohta K, Yamaguchi T, Matsubara T, Takahashi T, Nakajima T, Muto T, Ikari T, Yanagisawa A, Kato Y: Proliferative activity of intrahepatic colorectal metastases after preoperative hemihepatic portal vein embolization. Hepatology 2001;34:267-272.

21 Jaeck D, Oussoultzoglou E, Rosso E, Greget M, Weber JC, Bachellier P: A two-stage hepatectomy procedure combined with portal vein embolization to achieve curative resection for initially unresectable multiple and bilobar colorectal liver metastases. Ann Surg 2004;240:1037-1049, discussion 1049-1051.

22 Zorzi D, Laurent A, Pawlik TM, Lauwers GY, Vauthey JN, Abdalla EK: Chemotherapy-associated hepatotoxicity and surgery for colorectal liver metastases. Br J Surg 2007; 94:274-286.

-23 Vauthey JN, Pawlik TM, Ribero D, Wu TT, Zorzi D, Hoff PM, Xiong HQ, Eng C, Lauwers GY, Mino-Kenudson M, Risio M, Muratore A, Capussotti L, Curley SA, Abdalla EK: Chemotherapy regimen predicts steatohepatitis and an increase in 90-day mortality after surgery for hepatic colorectal metastases. J Clin Oncol 2006;24:20652072.

-24 Sugai Y, Komatani A, Hosoya T, Yamaguchi K: Response to percutaneous transhepatic portal embolization: new proposed parameters by ${ }^{99 \mathrm{~m}} \mathrm{Tc}$-GSA SPECT and their usefulness in prognostic estimation after hepatectomy. J Nucl Med 2000;41:421-425.

25 Li J, Girotti P, Königsrainer I, Ladurner R, Königsrainer A, Nadalin S: ALPPS in right trisectionectomy: a safe procedure to avoid postoperative liver failure? J Gastrointest Surg 2013;17:956-961.

-26 Ribero D, Abdalla EK, Madoff DC, Donadon M, Loyer EM, Vauthey JN: Portal vein embolization before major hepatectomy and its effects on regeneration, resectability and outcome. Br J Surg 2007;94:1386-1394. 